- Original Article

\title{
Health-Related Quality of Life and Cardiovascular Disease Risk in Korean Adults
}

\author{
Hyeon-Young Ko, Jung-Kwon Lee*, Jin-Young Shin, Euni Jo \\ Department of Family Medicine, Samsung Medical Center, Sungkyunkwan University School of Medicine, Seoul, Korea
}

Background: Health-related quality of life (HRQoL) is considered an important outcome measure in chronic diseases, in particular cardiovascular disease (CVD), which is known to be associated with impaired HRQoL. However, few studies have examined HRQoL in individuals at high risk of CVD.

Methods: Using the Fifth Korea National Health and Nutrition Examination Survey 2010-2012, we analyzed data from 10,307 adults aged $\geq 30$ years. The study subjects were stratified into 3 groups on the basis of their Framingham risk score - a 10-year estimate of CVD risk: $<10.0 \%$ (low risk), 10.0\%-19.9\% (moderate risk), and $\geq 20.0 \%$ (high risk). The EuroQol-5D (EQ-5D) was used to evaluate HRQoL.

Results: A significantly higher proportion of high-risk subjects than low-risk participants had impaired HRQoL (defined as the lowest quartile of the EQ-5D index); this held true even after adjustment for confounding factors in multivariable logistic regression analysis (men: odds ratio [OR], 1.62; 95\% confidence interval [CI], 1.24-2.11; women: $\mathrm{OR}, 1.46$; $95 \% \mathrm{CI}, 1.02-2.08$ ). In terms of the $5 \mathrm{EQ}-5 \mathrm{D}$ dimensions, a 10 -year CVD risk $\geq 20.0 \%$ was significantly associated with self-reported problems of mobility in men (OR, 3.15; 95\% CI, 2.02-4.90), and of mobility (OR, 1.56; 95\% CI, 1.09-2.24), self-care (OR, 2.14; 95\% CI, 1.09-4.22), and usual activity problems (OR, 1.80; 95\% CI, 1.17-2.78) in women.

Conclusion: A high CVD risk is associated with impaired HRQoL. After adjustment for demographic and clinical factors, a 10-year CVD risk $\geq 20.0 \%$ is an independent predictor of impaired HRQoL in the general population; in particular, of mobility problems in men, and of mobility, self-care, and usual activity problems in women.

Keywords: Cardiovascular Diseases; Risk Assessment; Quality of Life 


\section{INTRODUCTION}

Cardiovascular disease (CVD) is a leading cause of death and disability, as well as a major public health burden worldwide. ${ }^{1)}$ For this reason, primary care prevention of CVD is important. To this end, a comprehensive approach should be taken to the management of CVD risk factors; this would include lifestyle counseling, weight control, cholesterol-lowering medication, and blood pressure monitoring. ${ }^{2)}$

On the other hand, traditional outcome measures such as morbidity and mortality are insufficient in evaluating the benefits of medical interventions for chronic diseases like CVD; ${ }^{3)}$ this is because individuals at risk of CVD may be asymptomatic, or their symptoms may manifest only after a time. Moreover, the traditional outcome measures do not reflect functional ability, psychological status, and social interaction. ${ }^{4)}$

Health-related quality of life (HRQoL) is a broad, multifactorial concept that includes self-reported measures of physical and mental health. ${ }^{5)}$ It is considered particularly sensitive as an outcome measure of interventions and treatments in patients with established CVD. ${ }^{3)}$ Several studies have reported the relevance of HRQoL to CVD; for instance, Xie et al. ${ }^{6}$ found that coronary heart disease (CHD) is significantly associated with impaired HRQoL. In addition, several studies have shown that an increase in the number of CVD risk factors in CHD patients is associated with a gradual decrease in HRQoL. ${ }^{7,8)}$

Although CVD patients are known to have an impaired HRQoL, only a few studies have examined HRQoL in individuals at high risk of CVD. Among these, Ludt et al. ${ }^{9)}$ found that HRQoL is impaired in patients at risk of CVD, and that it is correlated with patient characteristics that have limited practical relevance. However, the study did not stratify the participants in terms of CVD risk, so it was not possible to evaluate the effect of risk on quality of life. The objective of this study, then, was to examine the impact of CVD risk on HRQoL in the general population.

\section{METHODS}

\section{Study Subjects}

This study used data from the fifth Korean National Health and Nutritional Examination Survey 2010-2012 (KNHANES V). The KNHANES was a national program that assessed the health and nutritional status of adults and children in Korea; the program comprised 3 distinct parts: a health interview, a health examination, and a nutrition survey. The study used a complex, multi-stage probability sample design, whereby the sample represented the total non-institutionalized civilian population of Korea. The KNHANES V was conducted from January 2010 to December 2012. A total of 3,840 households in 2010, 3,840 households in 2011, and 3,254 households in 2012 were selected, and all members of those households aged 1 year and over were included in the survey. Ultimately, 10,938 individuals in 2010, 10,589 individuals in 2011, and 10,069 individuals in 2012 were sampled. The response rates of the selected population were $77.5 \%$ in $2010,76.1 \%$ in 2011 , and $80.0 \%$ in 2012 . We have obtained informed consent for all participants. ${ }^{10-12)}$

Of the 25,534 participants in the KNHANES V, we analyzed data from 17,292 aged 30 years and over. We excluded 3,822 participants who did not provide either the health questionnaire or the blood test. In addition, 3,163 participants were excluded because they had a history of (1) established CVD, including myocardial infarction, angina, or stroke; (2) cancerparticularly of the stomach, liver, colon, breast, or lung; (3) osteo- or rheumatic arthritis; (4) asthma; (5) liver cirrhosis; or (6) chronic renal failure because these diseases have clear deleterious effects on HRQoL. ${ }^{6,7,13-15)}$ In the end, 10,307 subjects were enrolled.

\section{Assessment of Cardiovascular Disease Risk}

Although the Framingham risk score (FRS) has existed in various versions since it was first introduced, we used the variety described by D'Agostino et al., ${ }^{16)}$ which was designed for use in primary care. FRS is calculated using the following variables: age, sex, systolic blood pressure (SBP), antihypertensive medication, total cholesterol level, high density lipoprotein cholesterol level, current smoking status, and diabetes mellitus. The version we used provides the relative risk over the next 10 years -comparing the FRS of the subject with the estimated average risk in the same age and sex group. We calculated the 10-year risk of a cardiovascular event on the basis of FRS, and accordingly divided our study subjects into 3 groups: < 10.0\% (low risk), $10.0 \%-19.9 \%$ (moderate risk), and $\geq 20.0 \%$ (high risk).

Subjects were considered diabetic when their fasting glucose was $\geq 126 \mathrm{mg} / \mathrm{dL}$, when insulin or oral hypoglycemic medications were being used, when the subject self-reported diagnosis by a doctor, or when hemoglobin Alc (HbAlc) was $\geq 6.5 \%$; however, the last criterion was used in the 2011 and 2012 groups only, because HbAlc had not been measured in 2010. SBP was measured 3 times in each subject, and we used the mean of the second and third measurements. Information regarding antihypertension medication was based on the health questionnaire, and SBP treatment was defined as taking an antihypertension drug on more than 20 days per month. Smoking status was also determined using the questionnaire; current smokers included both those who smoked every day at the time of the survey and those who smoked intermittently at that time. All items were assessed via interview with trained staff.

\section{Measurement of Health-Related Quality of Life}

The EuroQol-5D (EQ-5D) index was used to evaluate HRQoL. ${ }^{17)}$ The EQ-5D records the levels of self-reported problems in 5 dimensions (mobility, self-care, usual activities, pain/ 
discomfort, and anxiety/depression). Each of the dimensions is assessed on the basis of single question with 3 response levels: 'no problems,' 'some problems,' and 'extreme problems.' The combinations of the responses to the 5 questions are converted into $243\left(3^{5}\right)$ health states, which are then transformed into a utility score, which in our study was weighted according to the Korean value set. ${ }^{18)} \mathrm{EQ}-5 \mathrm{D}$ scores range from -0.171 to 1 , where 1 indicates no problems in any of the 5 dimensions, 0 indicates death, and negative values indicate a health status worse than death.

\section{Covariates}

Self-reported questionnaires were used to assess the demographic and clinical characteristics of the participants. Income levels were divided into 4 groups on the basis of the average monthly household wage: lowest quartile, lower, higher, and highest quartile. Educational level was categorized as 'elementary or lower,' 'middle school,' 'high school,' or 'university or higher.' Employment status was classified as 'employed' or 'unemployed,' regardless of the type of job. Marital status was divided into 3 categories: 'married living with partner,' 'never married', and 'married not living with partner' (divorced, widowed, or separated). Subjects were asked to provide information on their level of psychological stress (none, low, moderate, and extreme), and stress level was classified binarily as 'none/low' or 'moderate/extreme.' Alcohol consumption was assessed on the basis of drinking frequency, and participants were divided into 2 groups in this regard: $\geq 2$ drinks/wk and $<2$ drinks/wk. In addition, subjects were divided into 4 groups in terms of exercise: 'vigorous,' 'moderate,' and 'low-intensity' exercise, as well as 'no exercise.' Vigorous exercise was defined as $>20$ minutes, $\geq 3$ times per week, of jogging, climbing, high speed cycling or swimming, soccer, basketball, jump rope, squash, tennis (singles), or manual labor involving heavy loads. Moderate exercise was defined as $>30$ minutes, $\geq 5$ times per week, of low speed swimming, tennis (doubles), volleyball, badminton, table tennis, or manual labor involving light loads. Low intensity exercise was defined as walking or commuting for $>30$ minutes, $\geq 5$ times per week. Body mass index (BMI) was categorized as $<18.5$, $18.5-23.0,23.0-24.9$, and $\geq 25.0 \mathrm{~kg} / \mathrm{m}^{2}$. Menopausal status, including both natural and artificial menopause, was considered in women, because menopause causes a decrease in quality of life that is independent of age and other socio-demographic variables. ${ }^{19)}$

\section{Statistical Analysis}

All estimates were calculated using the appropriate sampling weight provided in the KNHANES V; standard errors (SEs) were calculated in all analyses to reflect the complex design and weight of the survey to obtain a representative of the Korean population. Continuous data are expressed as mean $\pm \mathrm{SE}$ and were assessed using one way analysis of variance among the three 10-year risk groups ('low-,' 'moderate-,' and 'high-risk'). Categorical data were expressed as percentages and assessed using the chi-square test among the 3 risk groups. All analyses were conducted separately for men and women.

To evaluate the effect of CVD risk on the EQ-5D dimensions, we consolidated the 3 levels of answers into 2 groups: 'problem absent' or 'problem present.' The 'problem present' group included those who responded 'some problem' or 'extreme problem' among the 3 levels of severity. The proportions of participants reporting problems, either 'some problem' or 'extreme problem, in the 3 groups were assessed using the chi-square test to evaluate trends regarding each EQ-5D dimension.

Because of the inherent non-normal distribution of the EQ5 D index, we defined impaired HRQoL as the lowest quintile of EQ-5D index score and performed a univariable logistic regression analysis to assess the relationship between impaired HRQoL and clinical or demographic data; for this purpose, subjects were divided into 3 age groups: $30-44,45-64$, and $\geq 65$ years old.

Next, to assess whether a 10-year risk of CVD was independently associated with HRQoL, we constructed multivariable logistic regression models that had been adjusted for other potential confounding variables. Parameters that had shown significant association in the univariable analysis, or that were of considerable theoretical relevance, were entered into the multivariable analysis. Because calculation of the 10-year CVD risk involves comparing the individual's estimated risk to the average of the same age and sex group, age was not adjusted for in the multivariable logistic regression.

All analyses were conducted using IBM SPSS software for Windows ver. 21.0 (IBM Co., Armonk, NY, USA), and a 2-sided P-value $<0.05$ was deemed statistically significant. The study protocol was approved by the institutional review board of the Samsung Medical Center (SMC 2014-08-095).

\section{RESULTS}

In total, 4,828 men and 5,479 women were included in the study. In men, the proportions of low-, moderate-, and high-risk subjects were $47.1 \%, 22.7 \%$, and $30.2 \%$, respectively; in women, the proportions were $80.8 \%, 13.5 \%$, and $5.7 \%$. The characteristics of the subjects, stratified on the basis of 10-year CVD risk, are shown in Table 1. In both men and women, household income, years of education, and employment rate were lower in highrisk subjects. Concerning comorbidities, the high-risk group tended to have greater rates of hypertension, dyslipidemia, and diabetes mellitus; this held true for both sexes.

The threshold of the lowest EQ-5D quintile was at 1.000 in men and at 0.913 in women. In men, the proportions of subjects with impaired HRQoL in the low-, moderate-, and highrisk groups were $16.3 \%, 21.7 \%$, and $31.7 \%$, respectively; in wom- 
Table 1. Study population classified on the basis of 10-year CVD risk and stratified by sex

\begin{tabular}{|c|c|c|c|c|c|c|c|c|}
\hline \multirow{3}{*}{ Variable } & \multicolumn{8}{|c|}{ 10-Year CVD risk } \\
\hline & \multicolumn{4}{|c|}{ Men $(\mathrm{N}=4,828)$} & \multicolumn{4}{|c|}{ Women $(N=5,479)$} \\
\hline & $<10.0 \%$ & $10.0 \%-19.9 \%$ & $\geq 20.0 \%$ & P-value & $<10.0 \%$ & $10.0 \%-19.9 \%$ & $\geq 20.0 \%$ & P-value \\
\hline Total & $2,273(47.1)$ & $1,095(22.7)$ & $1,460(30.2)$ & & $4,425(80.8)$ & 740 (13.5) & $314(5.7)$ & \\
\hline Age (y) & $40.5 \pm 0.2$ & $52.6 \pm 0.3$ & $62.3 \pm 0.3$ & $<0.001$ & $44.0 \pm 0.2$ & $63.4 \pm 0.5$ & $70.6 \pm 0.6$ & $<0.001$ \\
\hline Quartile of household income & & & & $<0.001$ & & & & $<0.001$ \\
\hline Lowest & 6.5 & 11.9 & 28.2 & & 9.0 & 35.1 & 48.1 & \\
\hline Lower intermediate & 27.1 & 24.3 & 27.8 & & 29.0 & 27.6 & 22.8 & \\
\hline Higher intermediate & 34.1 & 31.4 & 22.0 & & 31.6 & 20.7 & 14.6 & \\
\hline Highest & 32.3 & 32.3 & 22.1 & & 30.4 & 16.6 & 14.5 & \\
\hline Education level & & & & $<0.001$ & & & & $<0.001$ \\
\hline Elementary or lower & 3.6 & 16.5 & 32.3 & & 12.5 & 62.9 & 80.8 & \\
\hline Middle school & 6.7 & 15.6 & 19.5 & & 10.7 & 13.7 & 9.2 & \\
\hline High school & 37.6 & 37.9 & 32.6 & & 41.6 & 18.4 & 8.6 & \\
\hline University or higher & 52.1 & 30.0 & 15.6 & & 35.2 & 4.9 & 1.4 & \\
\hline Employed & 93.4 & 87.2 & 69.0 & $<0.001$ & 55.9 & 44.4 & 34.0 & $<0.001$ \\
\hline Marital status & & & & $<0.001$ & & & & $<0.001$ \\
\hline Married & 79.8 & 89.4 & 90.6 & & 86.2 & 65.1 & 51.3 & \\
\hline Single & 17.4 & 4.2 & 2.7 & & 5.7 & 0.7 & 0.1 & \\
\hline Divorced/widow/widower & 2.8 & 6.4 & 6.7 & & 8.1 & 34.2 & 48.6 & \\
\hline Current smoker & 44.4 & 50.6 & 56.7 & $<0.001$ & 5.9 & 6.4 & 6.6 & 0.84 \\
\hline Alcohol consumption ( $\geq 2 /$ wk) & 39.5 & 48.1 & 44.7 & $<0.001$ & 11.3 & 7.6 & 2.7 & $<0.001$ \\
\hline Physical activity & & & & 0.13 & & & & 0.02 \\
\hline No exercise & 50.3 & 51.2 & 50.6 & & 55.5 & 58.8 & 63.6 & \\
\hline Low-intensity & 26.1 & 26.1 & 26.9 & & 26.4 & 27.2 & 25.2 & \\
\hline Moderate to vigorous-intensity & 23.6 & 22.7 & 22.6 & & 18.1 & 14.0 & 11.2 & \\
\hline Level of stress (moderate or extreme) & 27.7 & 25.6 & 17.5 & $<0.001$ & 27.6 & 24.3 & 26.7 & 0.29 \\
\hline \multicolumn{9}{|l|}{ Comorbidity* } \\
\hline Hypertension & 4.5 & 21.2 & 47.1 & $<0.001$ & 6.8 & 53.7 & 73.3 & $<0.001$ \\
\hline Diabetes mellitus & 2.4 & 13.1 & 37.2 & $<0.001$ & 3.1 & 23.5 & 53.4 & $<0.001$ \\
\hline Dyslipidemia & 4.6 & 10.9 & 14.8 & $<0.001$ & 5.3 & 21.0 & 23.3 & $<0.001$ \\
\hline Depression & 1.1 & 3.0 & 1.4 & 0.006 & 4.6 & 7.8 & 2.3 & 0.002 \\
\hline Body mass index $\left(\mathrm{kg} / \mathrm{m}^{2}\right)$ & $24.2 \pm 0.1$ & $24.5 \pm 0.1$ & $24.3 \pm 0.1$ & 0.18 & $23.2 \pm 0.1$ & $24.6 \pm 0.2$ & $24.7 \pm 0.2$ & $<0.001$ \\
\hline \multicolumn{9}{|l|}{ Menopausal status } \\
\hline Premenopausal & - & - & - & & $3,020(98.4)$ & $46(1.5)$ & $2(0.1)$ & \\
\hline Postmenopausal & - & - & - & & $1,385(58.0)$ & $693(29.0)$ & 311 (13.0) & \\
\hline
\end{tabular}

Values are presented as number (\%), mean \pm standard error, or \%. Continuous data were assessed using one-way analysis of variance, while categorical data were assessed using the chi-square test among the 3 risk groups.

CVD, cardiovascular disease.

*Subjects reported whether they had ever been diagnosed with comorbidities by a doctor.

en, the proportions were $16.3 \%, 37.6 \%$, and $50.0 \%$. In the highrisk group, the rate of impaired HRQoL was higher in both men and women.

In the univariable logistic regression analysis, ORs were higher in the moderate-risk group (OR, 1.42; 95\% CI, 1.13-1.78; $\mathrm{P}=$ 0.002 in men; OR, 3.10; 95\% CI, 2.53-3.79; $\mathrm{P}<0.001$ in women) and the high-risk group (OR, 2.38; 95\% CI, 1.95-2.91; $\mathrm{P}<0.001$ in men; OR, 5.14; 95\% CI, 3.85-3.79; $\mathrm{P}<0.001$ in women) than in the low-risk group (Table 2). This association was attenuated but remained significant after adjustment for socioeconomic factors (household income, education, employed status, and marital status), psychological factors (stress level), health-related behaviors (alcohol intake and physical activity), menopausal status (only women), and BMI. In multivariable analysis, a 10 -year CVD risk $\geq 20.0 \%$ was an independent risk factor for impaired health utility in both sexes (OR, 1.62; 95\% CI, 1.24-
2.11; $\mathrm{P}<0.001$ in men; $\mathrm{OR}, 1.46 ; 95 \% \mathrm{CI}, 1.02-2.08 ; \mathrm{P}=0.04$ in women) (Tables 3,4 ).

Table 3 shows the association between 10-year CVD risk and the $5 \mathrm{EQ}-5 \mathrm{D}$ dimensions in men; in the multivariable logistic regression analysis a 10 -year CVD risk $\geq 20.0 \%$ was significantly associated with self-reported mobility problems (OR, 3.15; $95 \%$ CI, 2.02-4.90).

Table 4 shows the association between 10-year CVD risk and the $5 \mathrm{EQ}-5 \mathrm{D}$ dimensions in women; a 10-year CVD risk $\geq 20.0 \%$ was significantly associated with self-reported mobility (OR, 1.56; 95\% CI, 1.09-2.24), self-care (OR, 2.14; 95\% CI, 1.09-4.22) and usual activity problems (OR, 1.80; 95\% CI, 1.17-2.78).

\section{DISCUSSION}

We have demonstrated that a high CVD risk is significantly as- 
Table 2. Univariable logistic regression analysis to assess the relationship between impaired HRQoL ${ }^{*}$ and demographic or clinical factors

\begin{tabular}{|c|c|c|c|c|}
\hline \multirow{2}{*}{ Variable } & \multicolumn{2}{|c|}{ Men } & \multicolumn{2}{|c|}{ Women } \\
\hline & Unadjusted OR (95\% Cl) & P-value & Unadjusted OR (95\% Cl) & P-value \\
\hline \multicolumn{5}{|l|}{ Age $(y)$} \\
\hline $30-44$ & Reference & & Reference & \\
\hline $45-64$ & $1.48(1.20-1.81)$ & $<0.001$ & $1.87(1.56-2.24)$ & $<0.001$ \\
\hline$\geq 65$ & $3.31(2.62-4.16)$ & $<0.001$ & $7.18(5.74-8.97)$ & $<0.001$ \\
\hline \multicolumn{5}{|l|}{ Quartile of household income } \\
\hline Highest & Reference & & Reference & \\
\hline Higher intermediate & $1.09(0.85-1.39)$ & 0.51 & $1.19(0.93-1.51)$ & 0.16 \\
\hline Lower intermediate & $1.46(1.15-1.85)$ & 0.002 & $1.52(1.20-1.93)$ & 0.001 \\
\hline Lowest & $2.93(2.24-3.83)$ & $<0.001$ & $4.63(3.56-6.02)$ & $<0.001$ \\
\hline \multicolumn{5}{|l|}{ Education level } \\
\hline University or more & Reference & & Reference & \\
\hline High school & $1.29(1.04-1.61)$ & 0.02 & $1.31(1.02-1.68)$ & $<0.001$ \\
\hline Middle school & $1.99(1.51-2.62)$ & $<0.001$ & $1.87(1.40-2.50)$ & $<0.001$ \\
\hline Elementary or less & $3.24(2.54-4.14)$ & $<0.001$ & $5.22(4.16-6.55)$ & 0.04 \\
\hline Unemployed status & $2.64(2.13-3.27)$ & $<0.001$ & $1.47(1.28-1.73)$ & $<0.001$ \\
\hline \multicolumn{5}{|l|}{ Marital status } \\
\hline Married & Reference & & Reference & \\
\hline Single & $0.93(0.68-1.27)$ & 0.66 & $0.79(0.50-1.23)$ & 0.29 \\
\hline Divorced/widow/widower & $1.56(1.01-2.40)$ & 0.04 & $3.05(2.50-3.71)$ & $<0.001$ \\
\hline Current smoker & $1.16(0.98-1.38)$ & 0.08 & $1.68(1.22-2.30)$ & 0.001 \\
\hline Alcohol consumption ( $\geq 2 /$ wk) & $1.02(0.85-1.22)$ & 0.81 & $0.93(0.72-1.21)$ & 0.593 \\
\hline \multicolumn{5}{|l|}{ Physical activity } \\
\hline Moderate to vigorous-intensity & Reference & & Reference & \\
\hline Low-intensity & $1.01(0.77-1.32)$ & 0.94 & $1.04(0.80-1.35)$ & 0.79 \\
\hline No exercise & $1.19(0.95-1.49)$ & 0.13 & $1.34(1.05-1.69)$ & 0.02 \\
\hline Level of stress (moderate or extreme) & $2.19(1.84-2.61)$ & $<0.001$ & $2.81(2.40-3.30)$ & $<0.001$ \\
\hline \multicolumn{5}{|l|}{ Comorbidities $^{\dagger}$} \\
\hline Hypertension & $1.79(1.49-2.16)$ & $<0.001$ & $2.91(2.41-3.51)$ & $<0.001$ \\
\hline Diabetes mellitus & $1.46(1.17-1.81)$ & 0.001 & $2.09(1.63-2.66)$ & $<0.001$ \\
\hline Dyslipidemia & $1.79(1.37-2.32)$ & $<0.001$ & $1.98(1.55-2.53)$ & $<0.001$ \\
\hline Depression & $4.96(2.86-8.60)$ & $<0.001$ & $3.50(2.55-4.81)$ & $<0.001$ \\
\hline \multicolumn{5}{|l|}{ Body mass index $\left(\mathrm{kg} / \mathrm{m}^{2}\right)$} \\
\hline $18.5-22.9$ & Reference & & Reference & \\
\hline$<18.5$ & $1.25(0.72-2.16)$ & 0.43 & $1.57(1.07-2.30)$ & 0.02 \\
\hline 23-24.9 & $0.89(0.71-1.13)$ & 0.33 & $1.18(0.94-1.48)$ & 0.17 \\
\hline$\geq 25$ & $0.93(0.74-1.16)$ & 0.50 & $1.55(1.28-1.89)$ & $<0.001$ \\
\hline Postmenopausal status & - & & $3.04(2.60-3.54)$ & $<0.001$ \\
\hline \multicolumn{5}{|l|}{ 10-Year cardiovascular disease risk } \\
\hline$<10.0 \%$ & Reference & & Reference & \\
\hline $10.0 \%-19.9 \%$ & $1.42(1.13-1.78)$ & 0.002 & $3.10(2.53-3.79)$ & $<0.001$ \\
\hline$\geq 20.0 \%$ & $2.38(1.95-2.91)$ & $<0.001$ & $5.14(3.85-6.87)$ & $<0.001$ \\
\hline
\end{tabular}

$\mathrm{HRQ} \mathrm{L}$, health related quality of life; $\mathrm{OR}$, odds ratio; $\mathrm{Cl}$, confidence interval.

*Impaired HRQoL was defined as the lowest quintile of the EuroQoL-5D score. 'Subjects reported whether they had ever been diagnosed with comorbidities by a doctor.

Table 3. Multivariable logistic regression analysis to assess the relationship between impaired $H R Q L^{*}$ and the $E Q-5 D$ dimensions in men

\begin{tabular}{|c|c|c|c|c|c|c|}
\hline & \multirow{2}{*}{ Impaired HRQoL } & \multicolumn{5}{|c|}{ EQ-5D dimensions } \\
\hline & & Mobility & Self-care & Usual activity & Pain/discomfort & Anxiety/depression \\
\hline \multicolumn{7}{|c|}{ 10-Year cardiovascular disease risk } \\
\hline$<10.0 \%$ & Reference & Reference & Reference & Reference & Reference & Reference \\
\hline $10.0 \%-19.9 \%$ & $1.21(0.94-1.57)$ & $2.07^{\dagger}(1.31-3.29)$ & $2.69(0.99-7.31)$ & $1.67(0.90-3.11)$ & $1.03(0.76-1.39)$ & $1.67(1.11-2.53)$ \\
\hline$\geq 20.0 \%$ & $1.62^{\ddagger}(1.24-2.11)$ & $3.15^{\ddagger}(2.02-4.90)$ & $2.23(0.68-7.28)$ & $1.70(0.87-3.33)$ & $1.16(0.86-1.58)$ & $1.50^{\dagger}(0.92-2.45)$ \\
\hline
\end{tabular}

Values are presented as odds ratio (95\% confidence interval). The results were adjusted for household income, education level, employed status, marital status, stress level, alcohol intake, physical activity, and body mass index.

$H R Q D L$, health related quality of life; EQ-5D, EuroQoL-5D.

${ }^{*}$ Impaired HRQoL was defined as the lowest quintile of the $E Q-5 D$ score. ${ }^{\dagger} \mathrm{P}<0.05 .{ }^{\ddagger} \mathrm{P}<0.001$.

sociated with impaired HRQoL after adjusting for demographic and clinical factors. These findings suggest that HRQoL assess- ment might be useful in cardiovascular risk management.

Concerning the 5 dimensions of the EQ-5D, high CVD risk 
Table 4. Multivariable logistic regression analysis to assess the relationship between impaired HRQoL* and the EQ-5D in women

\begin{tabular}{|c|c|c|c|c|c|c|}
\hline & \multirow{2}{*}{ Impaired HRQoL } & \multicolumn{5}{|c|}{$E Q-5 D$} \\
\hline & & Mobility & Self-care & Usual activity & Pain/discomfort & Anxiety/depression \\
\hline \multicolumn{7}{|c|}{ 10-Year cardiovascular disease risk } \\
\hline$<10.0 \%$ & Reference & Reference & Reference & Reference & Reference & Reference \\
\hline $10.0 \%-19.9 \%$ & $1.25(0.98-1.60)$ & $1.33^{\dagger}(1.01-1.75)$ & $1.55(0.89-2.69)$ & $1.40(0.98-2.01)$ & $1.32^{\dagger}(1.03-1.69)$ & $0.83(0.60-1.15)$ \\
\hline$\geq 20.0 \%$ & $1.46^{\dagger}(1.02-2.08)$ & $1.56^{\dagger}(1.09-2.24)$ & $2.14^{\dagger}(1.09-4.22)$ & $1.80^{\ddagger}(1.17-2.78)$ & $1.08(0.77-1.52)$ & $0.75(0.45-1.25)$ \\
\hline
\end{tabular}

Values are presented as odds ratio (95\% confidence interval). The results were adjusted for household income, education level, employed status, marital status, stress level, alcohol intake, physical activity, body mass index, and menopausal status.

HRQoL, health related quality of life; EQ-5D, EuroQoL 5 dimensions.

${ }^{*}$ Impaired HRQoL was defined as the lowest quintile of the EQ-5D score. ${ }^{\mathrm{P}} \mathrm{P}<0.05 .{ }^{\mathrm{f} P}<0.001$

was significantly associated with mobility problems in both men and women, as well as with self-care and usual activity problems in women only. These findings are consistent with the results of previous studies involving patients with established CVD. For instance, in a 2008 study analyzing the national survey of the United States, the Physical Component Summary score of the 12-item Short Form Health Survey was significantly lower in all CHD subgroups than in those without CHD; however, the Mental Component Summary score was lower in some CHD subgroups only. ${ }^{6}$ Another study found that CHD seems to influence both the physical component and overall health, but has limited influence on mental health.7)

We also assessed the impact of socio-demographic predictors on impaired HRQoL using multivariable regression analysis. Impaired HRQoL was correlated with lower household income, lower educational level, unemployed status, not living with marriage partner, moderate or higher levels of stress, other comorbidities, and postmenopausal state. This is consistent with previous research; ${ }^{20)}$ all these factors are detrimental to HRQoL and increase CVD risk. ${ }^{21)}$ However, we noted in addition that a 10 -year CVD risk of $\geq 20 \%$ is an independent predictor of impaired HRQoL, even after correcting for such covariates. Thus, our findings suggest that an increased CVD risk is important in impaired HRQoL.

It is well known that socioeconomic status (SES) has a significant impact on quality of life. Nonetheless, comprehensive lifestyle changes can improve cardiovascular health in people of low SES to a similar degree as in people of high SES. ${ }^{22)}$ Put another way, even despite the effects of the SES, cardiovascular risk management may improve HRQoL.

We found that certain factors differ between men and women with regard to their influence on CVD risk. For example, in the 'moderate-risk' and 'high-risk' groups, the proportion of women who were divorced or widowed was much higher than that of men, probably because the average age of the men differed from that of the women in both groups. The proportions of our study subjects who were divorced or widowed were as follows: $5.0 \%$ of men $50-59$ years old, $5.9 \%$ of men $60-69$ years old, $25.3 \%$ of women $60-69$ years old, and $57.3 \%$ of women $70-$ 79 years old. In both men and women, these numbers are simi- lar to the 'divorced or widowed' rates at the average age in both the 'moderate risk' and 'high-risk' groups. Another apparent reason for the large difference in the 'divorced or widowed' rate is that most married women have a husband older than themselves in South Korea. In all risk groups, the sexes differed in terms of stress level; this is most likely due to age and post-menopausal status. Whereas the perceived stress level decreased with increasing age in men, the level in women decreased with increasing age as far as the ' $50-59$ years old' group, and increased after that. ${ }^{12)}$ Furthermore, psychological stress is known to be increased in peri- and post-menopausal women ${ }^{23)}$ Because more than $99 \%$ of women in the 'high-risk' group were postmenopausal, we consider menopause to have influenced the increased stress level in that group.

There may be several reasons high CVD risk has a negative impact on HRQoL. For example, previous studies have reported that the HRQoL of individuals with hypertension is worse than that of normotensive persons. ${ }^{24)}$ Those with diabetes or poorly controlled blood glucose have poorer HRQoL than those with normal glucose. ${ }^{25)}$ In addition, HRQoL is significantly associated with current smoking. ${ }^{26)}$ Regarding dyslipidemia, results differ as to whether it is associated with impaired HRQoL. Specifically, in a large hospital-based survey among individuals without $\mathrm{CHD}$, those with dyslipidemia had lower adjusted mean HRQoL scores, ${ }^{27)}$ whereas another study found no association. ${ }^{28)}$ In the present study, the 'high-risk' group had the highest rates of hypertension, dyslipidemia, diabetes mellitus, and current smoking. Future studies into the association of quality of life with CVD should address predictors and mechanisms acting on HRQoL.

The present study had several important strengths. The study involved a representative sample of the general Korean population, and the quality of KNHANES study procedures was rigorously controlled. Our findings have important public health implications for the general population.

On the other hand, several limitations of our study need to be noted. Firstly, as this study was conducted using cross-sectional data, it was not possible to assess the causal relationship between 10-year CVD risk and HRQoL. Furthermore, the EQ$5 \mathrm{D}$ instrument has been reported as showing a ceiling effect. ${ }^{29)}$ 
The highest scores (1.000) of the EQ-5D index in our study were $78.2 \%$ in men and $68.8 \%$ in women. The index may be less sensitive in describing mild-severity health levels. Nonetheless, the EQ-5D instrument is a reasonably valid and reliable instrument for surveying the general population. ${ }^{30)}$ The index may be useful in clinical research and epidemiological studies to generate preference-based HRQoL evaluations.

In conclusion, high CVD risk is an independent predictor of impaired HRQoL after adjustment for socio-demographic factors and certain medical comorbidities, namely mobility problems in men, and mobility, self-care, and usual activity problems in women. Therefore, clinicians should focus not only on controlling modifiable risk factors, but also on the subjective perception of health in individuals at high risk of CVD.

\section{CONFLICT OF INTEREST}

No potential conflict of interest relevant to this article was reported.

\section{REFERENCES}

1. Lozano R, Naghavi M, Foreman K, Lim S, Shibuya K, Aboyans V, et al. Global and regional mortality from 235 causes of death for 20 age groups in 1990 and 2010: a systematic analysis for the Global Burden of Disease Study 2010. Lancet 2012;380:2095-128.

2. Alvarez-Bueno C, Cavero-Redondo I, Martinez-Andres M, Arias-Palencia N, Ramos-Blanes R, Salcedo-Aguilar F. Effectiveness of multifactorial interventions in primary health care settings for primary prevention of cardiovascular disease: a systematic review of systematic reviews. Prev Med 2015;76 Suppl:S68-75.

3. Cepeda-Valery B, Cheong AP, Lee A, Yan BP. Measuring health related quality of life in coronary heart disease: the importance of feeling well. Int J Cardiol 2011;149:4-9.

4. Wenger NK, Mattson ME, Furberg CD, Elinson J. Assessment of quality of life in clinical trials of cardiovascular therapies. Am J Cardiol 1984; 54:908-13.

5. Centers for Disease Control and Prevention. Measuring healthy days: population assessment of health-related quality of life. Atlanta (GA): Centers for Disease Control and Prevention; 2000.

6. Xie J, Wu EQ, Zheng ZJ, Sullivan PW, Zhan L, Labarthe DR. Patient-reported health status in coronary heart disease in the United States: age, sex, racial, and ethnic differences. Circulation 2008;118:491-7.

7. De Smedt D, Clays E, Annemans L, Doyle F, Kotseva K, Pająk A, et al. Health related quality of life in coronary patients and its association with their cardiovascular risk profile: results from the EUROASPIRE III survey. Int J Cardiol 2013;168:898-903.

8. Sullivan PW, Ghushchyan V, Wyatt HR, Wu EQ, Hill JO. Impact of cardiometabolic risk factor clusters on health-related quality of life in the U.S. Obesity (Silver Spring) 2007;15:511-21.

9. Ludt S, Wensing M, Szecsenyi J, van Lieshout J, Rochon J, Freund T, et al. Predictors of health-related quality of life in patients at risk for cardiovascular disease in European primary care. PLoS One 2011;6:e29334.
10. Korea Centers for Disease Control and Prevention. Korean health statistics 2010: Korea National Health and Nutrition Examination Survey (KNHANES V-1). Cheongju: Korea Centers for Disease Control and Prevention; 2011.

11. Korea Centers for Disease Control and Prevention. Korean health statistics 2011: Korea National Health and Nutrition Examination Survey (KNHANES V-2). Cheongju: Korea Centers for Disease Control and Prevention; 2012.

12. Korea Centers for Disease Control and Prevention. Korean health statistics 2012: Korea National Health and Nutrition Examination Survey (KNHANES V-3). Cheongju: Korea Centers for Disease Control and Prevention; 2013.

13. Richardson LC, Wingo PA, Zack MM, Zahran HS, King JB. Health-related quality of life in cancer survivors between ages 20 and 64 years: population-based estimates from the Behavioral Risk Factor Surveillance System. Cancer 2008;112:1380-9.

14. Alonso J, Ferrer M, Gandek B, Ware JE Jr, Aaronson NK, Mosconi P, et al. Health-related quality of life associated with chronic conditions in eight countries: results from the International Quality of Life Assessment (IQOLA) Project. Qual Life Res 2004;13:283-98.

15. Lee H, Oh YJ, Kim M, Kim H, Lee JP, Kim S, et al. The association of moderate renal dysfunction with impaired preference-based healthrelated quality of life: third Korean national health and nutritional examination survey. BMC Nephrol 2012;13:19.

16. D'Agostino RB Sr, Vasan RS, Pencina MJ, Wolf PA, Cobain M, Massaro $\mathrm{JM}$, et al. General cardiovascular risk profile for use in primary care: the Framingham Heart Study. Circulation 2008;117:743-53.

17. Rabin R, de Charro F. EQ-5D: a measure of health status from the EuroQol Group. Ann Med 2001;33:337-43.

18. Nam H, Kim K, Kwon S, Koh K, Poul K. EQ-5D Korean valuation study using time trade of method. Cheongju: Korea Centers for Disease Control and Prevention; 2007.

19. Blumel JE, Castelo-Branco C, Binfa L, Gramegna G, Tacla X, Aracena B, et al. Quality of life after the menopause: a population study. Maturitas 2000;34:17-23.

20. Jiang Y, Hesser JE. Associations between health-related quality of life and demographics and health risks. Results from Rhode Island's 2002 behavioral risk factor survey. Health Qual Life Outcomes 2006;4:14.

21. Kaplan GA, Keil JE. Socioeconomic factors and cardiovascular disease: a review of the literature. Circulation 1993;88(4 Pt 1):1973-98.

22. Govil SR, Weidner G, Merritt-Worden T, Ornish D. Socioeconomic status and improvements in lifestyle, coronary risk factors, and quality of life: the Multisite Cardiac Lifestyle Intervention Program. Am J Public Health 2009;99:1263-70.

23. Bromberger JT, Meyer PM, Kravitz HM, Sommer B, Cordal A, Powell L, et al. Psychologic distress and natural menopause: a multiethnic community study. Am J Public Health 2001;91:1435-42.

24. Trevisol DJ, Moreira LB, Kerkhoff A, Fuchs SC, Fuchs FD. Health-related quality of life and hypertension: a systematic review and meta-analysis of observational studies. J Hypertens 2011;29:179-88.

25. Rubin RR, Peyrot M. Quality of life and diabetes. Diabetes Metab Res Rev 1999;15:205-18.

26. Tillmann M, Silcock J. A comparison of smokers' and ex-smokers' healthrelated quality of life. J Public Health Med 1997;19:268-73.

27. Lalonde L, Clarke AE, Joseph L, Mackenzie T, Grover SA; Canadian 
Collaborative Cardiac Assessment Group. Health-related quality of life with coronary heart disease prevention and treatment. J Clin Epidemiol 2001;54:1011-8.

28. Lalonde L, O'Connor A, Joseph L, Grover SA; Canadian Collaborative Cardiac Assessment Group. Health-related quality of life in cardiac patients with dyslipidemia and hypertension. Qual Life Res 2004;13:793804.
29. Bharmal M, Thomas J 3rd. Comparing the EQ-5D and the SF-6D descriptive systems to assess their ceiling effects in the US general population. Value Health 2006;9:262-71.

30. Kontodimopoulos N, Pappa E, Niakas D, Yfantopoulos J, Dimitrakaki C, Tountas Y. Validity of the EuroQoL (EQ-5D) instrument in a Greek general population. Value Health 2008;11:1162-9. 